\title{
Event Coreference Resolution by Iteratively Unfolding Inter-dependencies among Events
}

\author{
Prafulla Kumar Choubey and Ruihong Huang \\ Department of Computer Science and Engineering \\ Texas A\&M University \\ (prafulla.choubey, huangrh) dtamu.edu
}

\begin{abstract}
We introduce a novel iterative approach for event coreference resolution that gradually builds event clusters by exploiting inter-dependencies among event mentions within the same chain as well as across event chains. Among event mentions in the same chain, we distinguish within- and cross-document event coreference links by using two distinct pairwise classifiers, trained separately to capture differences in feature distributions of within- and crossdocument event clusters. Our event coreference approach alternates between WD and $\mathrm{CD}$ clustering and combines arguments from both event clusters after every merge, continuing till no more merge can be made. And then it performs further merging between event chains that are both closely related to a set of other chains of events. Experiments on the $\mathrm{ECB}+$ corpus show that our model outperforms state-of-the-art methods in joint task of WD and CD event coreference resolution.
\end{abstract}

\section{Introduction}

Event coreference resolution is the task of identifying event mentions and clustering them such that each cluster represents a unique real world event. The capability of resolving links among coreferring event identities is vital for information aggregation and many NLP applications, including topic detection and tracking, information extraction, question answering and text summarization (Humphreys et al., 1997; Allan et al., 1998; Daniel et al., 2003; Narayanan and Harabagiu, 2004; Mayfield et al., 2009; Zhang et al., 2015). Yet, studies on event coreference are few com- pared to the well-studied entity coreference resolution.

Event mentions that refer to the same event can occur both within a document (WD) and across multiple documents (CD). One common practice (Lee et al., 2012) to approach CD coreference task is to resolve event coreference in a megadocument created by concatenating topic-relevant documents, which essentially does not distinguish WD and CD event links.

However, intuitively, recognizing CD coreferent event pairs requires stricter evidence compared to WD event linking because it is riskier to link two event mentions from two distinct documents rather than the same document. In a perfect scenario where all WD event mentions are properly clustered and their participants and arguments are combined within a cluster, CD clustering can be performed with ease as sufficient evidences are collected through initial WD clustering. Therefore, another very common practice for event coreference is to first group event mentions within a document and then group WD clusters across documents (Yang et al., 2015).

Nonetheless, WD coreference chains are equally hard to resolve. Event mentions in the same document can look very dissimilar ("killed/ VB" and "murder/ NN"), have event arguments (i.e., participants and spatio-temporal information of an event (Bejan and Harabagiu, 2010)) partially or entirely omitted, or appear in distinct contexts compared to their antecedent event mentions, partially to avoid repetitions. Under this irresolute state, approaching WD and CD individually is incompetent.

While CD coreference resolution is overall difficult, we observe that some CD coreferent event mentions, especially the ones that appear at the beginning of documents, share sufficient contexts and are relatively easier to resolve. At the same 
time, many of them bear sufficient differences that can bring in new information and further lead to more WD merges and consequently more $\mathrm{CD}$ merges.

Guided by these observations, we present an event coreference approach that exploits interdependencies among event mentions within an event chain both within a document and across documents, by sequentially applying WD and CD merges in an alternating manner until no more merge can be made. We combine argument features of event mentions after each CD (WD) merge in order to resolve more difficult WD (CD) merges in the following iterations. Furthermore, our model uses two distinct pairwise classifiers that are separately trained with features intrinsic to each type. Specifically, the WD classifier uses features based on event mentions and their arguments while the CD classifier relies on features characterizing surrounding contexts of event mentions as well.

We further exploit second-order interdependencies across event clusters in order to resolve additional WD and CD coreferent event pairs. Intuitively, if two event mentions are related to the same set of events, it is likely that the two event mentions refer to the same real world event, even when their word forms and local contexts are distinct. Specifically, we merge event clusters if their event mentions are tightly associated (i.e., having the same dependency relations) or loosely associated (i.e., co-occurring in the same sentential context) with enough (i.e., passing a threshold) other events that are known coreferent.

Experimental results on the benchmark event coreference dataset, $\mathrm{ECB}+($ Cybulska and Vossen, $2014 \mathrm{~b}$,a), show that our model extensively exploits inter-dependencies between events and outperforms the state-of-the-art methods for both WD and $\mathrm{CD}$ event coreference resolution.

\section{Related Work}

Different approaches, focusing on either of WD or $\mathrm{CD}$ coreference chains, have been proposed for event coreference resolution. Works specific to WD event coreference includes pairwise classifiers (Ahn, 2006; Chen et al., 2009) graph based clustering method (Chen and Ji, 2009), information propagation (Liu et al., 2014), and markov logic networks Lu et al. (2016). As to only CD event coreference, Cybulska and Vossen (2015a) created pairwise classifiers using features indicating granularities of event slots and in another work (2015b), grouped events based on compatibilities of event contexts.

Like this work, several studies have considered both WD and CD event coreference resolution task together. However to simplify the problem, they (Lee et al., 2012; Bejan and Harabagiu, $2010,2014)$ created a meta-document by concatenating topic-relevant documents and treated both as an identical task. Most recently, Yang et al. (2015) applied a two-level clustering model that first groups event mentions within a document and then groups WD clusters across documents in a joint inference process. Our approach advances these works and emphasizes on different natures of WD and CD clusters along with the benefits of distinguishing WD merges from $\mathrm{CD}$ merges and exploiting their mutual dependencies.

Iterative models, in general, have been applied to both entity coreference resolution (Singh et al., 2009; Clark and Manning, 2015, 2016; Wiseman et al., 2016) and prior event coreference resolution (Lee et al., 2012) works, which gradually build clusters and enable later merges to benefit from earlier ones. Especially, Lee et al. (2012) used an iterative model to jointly build entity and event clusters and showed the advantages of information flow between entity and event clusters through semantic role features. Our model, by alternating between WD and CD merges, allows the multi-level flow of first order interdependencies. Moreover, additional cross cluster merges based on 2 nd order interdependencies effectively exploits the semantic relations among events, in contrast to only semantic roles (between events and arguments) used in previous work.

\section{System Overview and A Worked Example}

Inter-dependencies among event mentions can be effectively exploited by conducting sequential WD and CD merges in an iterative manner. In addition, recognizing second order relations between event chains relies on adequate number of event mentions that are already linked. Therefore, our model conducts event coreference in two stages. In the first stage, it iteratively conducts WD and CD merges as suggested by pairwise WD and CD merging classifiers respectively. Argument features of individual event mentions are propagated 


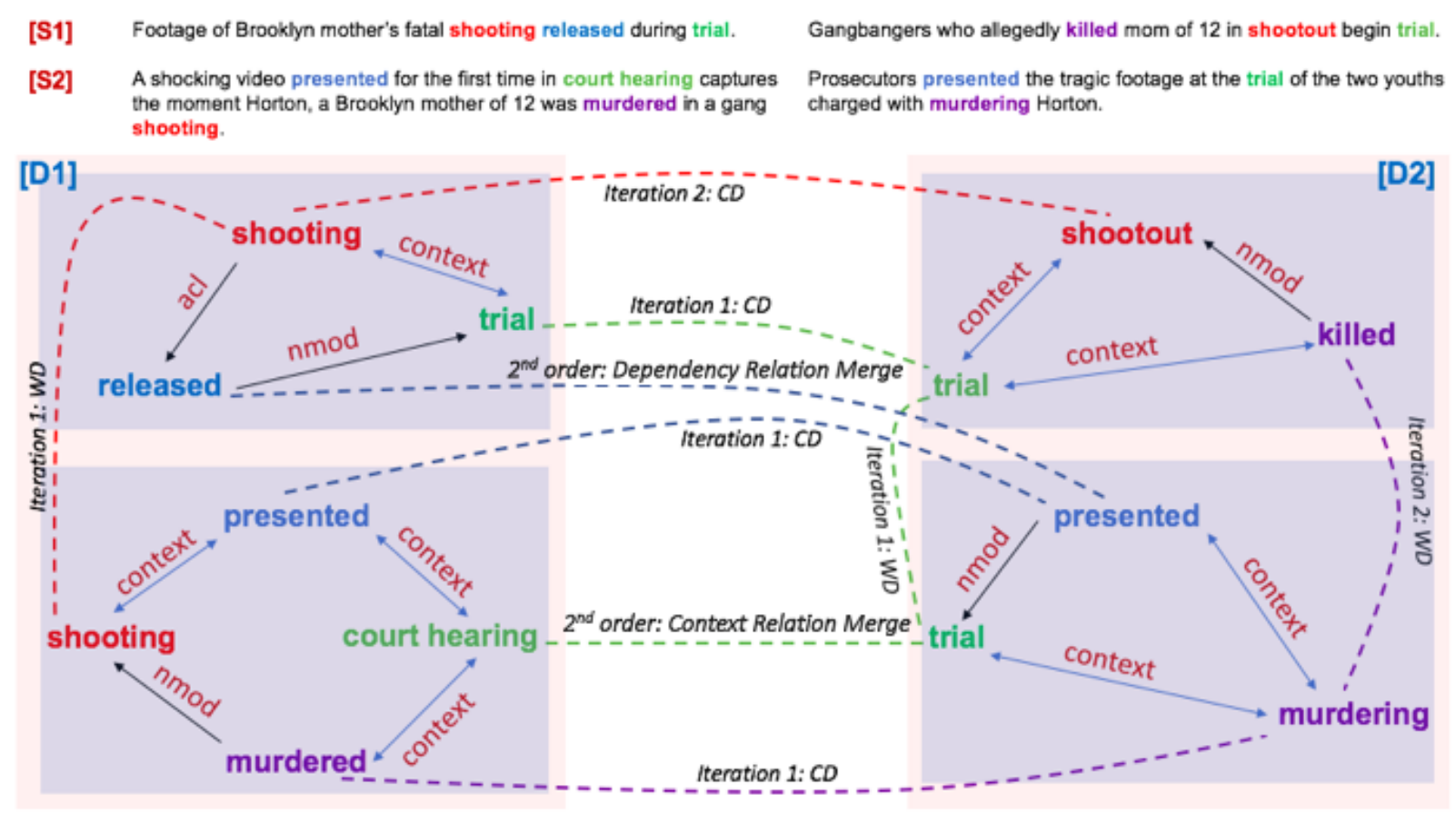

Figure 1: An example of Event Coreference using the iterative two stage model. All event mentions are boldfaced; solid arrow line between event mentions show second order relations between them; dashed lines link coreferent event mentions and are tagged with the type of merge.

within a cluster after each merge operation. In the second stage, it explores second order relations across event clusters w.r.t context event mentions in order to carefully generate candidate event clusters and perform further merging.

The example in Figure 1 illustrates the two stages of our proposed approach. It shows two iterations of $\mathrm{WD}$ and $\mathrm{CD}$ merges. In iteration 1 , relatively easy coreferent event mentions were linked, including the two shooting and two trial event mentions in $d o c_{1}$ and $d o c_{2}$ as well as the event mentions presented, trial and murder across the two documents. Argument propagation was conducted after each merge and murder's argument "mother of 12" in $d o c_{1}$ is combined with the murder event in $d o c_{2}$ after iteration 1. Then in iteration 2, more merges were made by recognizing additional coreferent event mentions including event mentions in one document (e.g., murdering and killed in $d o c_{2}$ ) and event mentions across the two documents (e.g., shooting in $d o c_{1}$ and shootout in $d o c_{2}$ ). Next, two additional merges were made by leveraging second-order inter-dependencies. Specifically, both the event mentions released in $d o c_{1}$ and presented in $d o c_{2}$ are in the same dependency relation ("nmod") with a mention of the trial event cluster, therefore, a new merge was made between clusters containing the two mentions. Following this, the event mentions court hearing in $d o c_{1}$ and trial in $d o c_{2}$ were identified to have multiple coreferent events in their sentential contexts, therefore, the clusters containing these two event mentions were merged as well.

\section{Detailed System Description: Exploiting Interdependencies between Events}

\subsection{Document Clustering}

Our approach starts with a pre-processing step that clusters input documents $(\mathcal{D})$ into a set of document clusters $(\mathcal{C})$. This is meant to reduce search space and mitigate errors (Lee et al., 2012). In our experiments, we used the Affinity Propagation algorithm (Buitinck et al., 2013) on $t f-i d f$ vectors, where terms are only proper nouns and verbs (excludes reporting and auxiliary verbs) in the document. While it is interesting to understand the influences of wrong document clusters to event coreference, this algorithm yielded perfect document clusters on the benchmark ECB+ 
dataset (Cybulska and Vossen, 2014b,a). This is consistent with the prior study (Lee et al., 2012) on the related ECB dataset (Bejan and Harabagiu, 2010) ${ }^{1}$, which shows that document clustering in the ECB dataset is trivial.

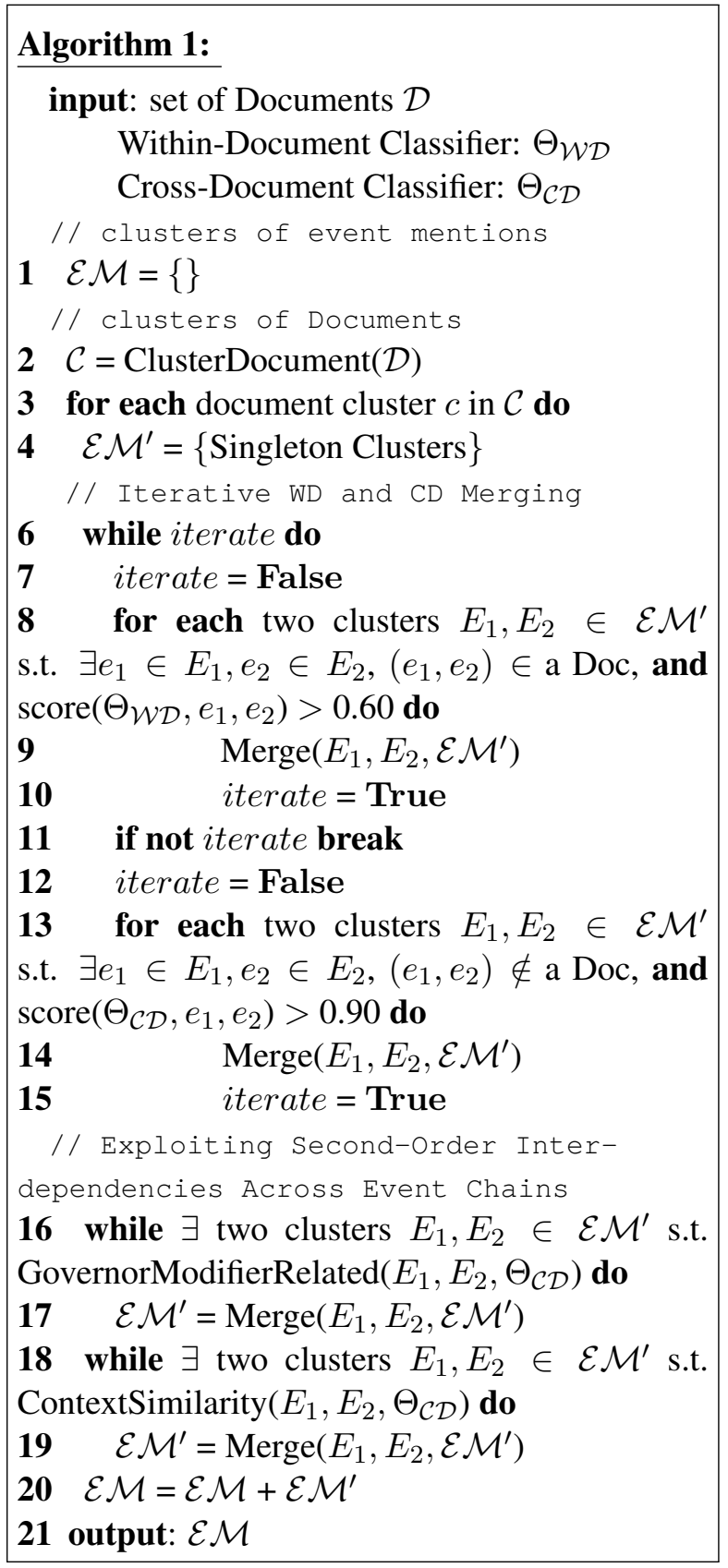

\subsection{Iterative WD and CD Merging}

We iteratively conduct WD merges and CD merges until no more merge can be done. We train pairwise classifiers for identifying event clusters to merge. Specifically for WD merges as indicated in lines 8-10 in Algorithm 1, we iteratively go through pairs of clusters that contain a pair

\footnotetext{
${ }^{1}$ The ECB+ dataset is an extended version of the ECB dataset. Both datasets have documents for the same 43 topics.
}

of within-document event mentions, one mention from each cluster. If the similarity score between the two event mentions is above a tuned threshold of $0.6^{2}$, we merge the two clusters. Similarly, for CD merges described in lines 13-15 of Algorithm 1 , we iteratively go through pairs of clusters that contain a pair of cross-document event mentions and merge the two clusters if the similarity score between the two event mentions is above another tuned threshold of $0.9^{3}$. Following each cluster pair merge, arguments are combined for the two merged clusters.

\subsection{Merging by Exploiting Second-Order Inter-dependencies Across Event Chains}

Intuitively, two event mentions that share events in their contexts are likely to be coreferent. Similarly, if their context events are coreferent, the two events are likely to be coreferent as well.

First, if two event mentions are in the same dependency relation with two other event mentions that are known coreferent, then the first two event mentions are likely to describe the same real world event as well. In steps 16-17 of Algorithm 1, we perform event cluster merges by collecting evidence pertaining to dependency relations. The subroutine Governor ModifierRelated $\left(E_{1}, E_{2}, \Theta_{\mathcal{C D}}\right)$ checks whether two event mentions $e_{1}$ and $e_{2}$, from clusters $E_{1}$ and $E_{2}$ respectively, have a related event $e_{3}$ from another cluster $E_{3}$, such that $E_{3} \notin\left\{E_{1}, E_{2}\right\}$ and pairs $\left(e_{1}, e_{3}\right),\left(e_{2}, e_{3}\right)$ are linked with the same dependency relation. Note that observing shared event mentions in the contexts will increase the likelihood that the two event mentions are coreferent, but we can not sufficiently infer the coreference relation yet, we still need to look at features describing the event mentions. Therefore, if the condition was satisfied, the subroutine eventually makes merges based on the $\mathrm{CD}$ confidence score assigned to the event pair $\left(e_{1}, e_{2}\right)$ but using a lower threshold of 0.8 .

In addition, seeing coreferent event mentions in the sentential contexts of two events will increase the likelihood that the two events are coreferent as well. Then as shown in steps $18-19$, we fur-

\footnotetext{
${ }^{2}$ all tunings are performed on Validation dataset (topics 23-25)

${ }^{3}$ Note that these high threshold for WD- and CD- classifiers are meant to retain high precision and avoid error propagation in subsequent stages. Output from each classifier is a number bounded in $[0,1]$.
} 


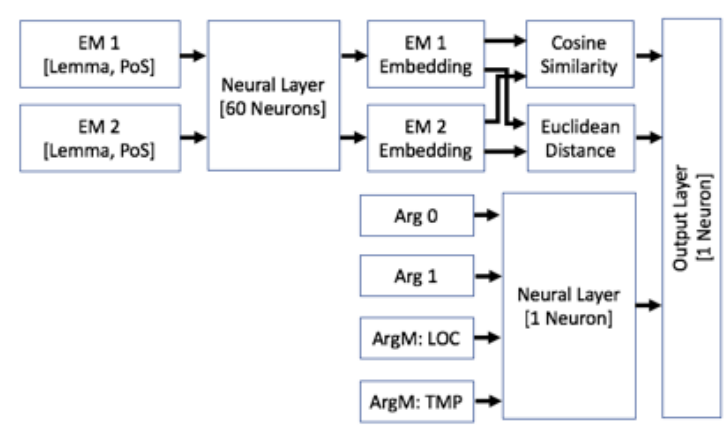

(a)

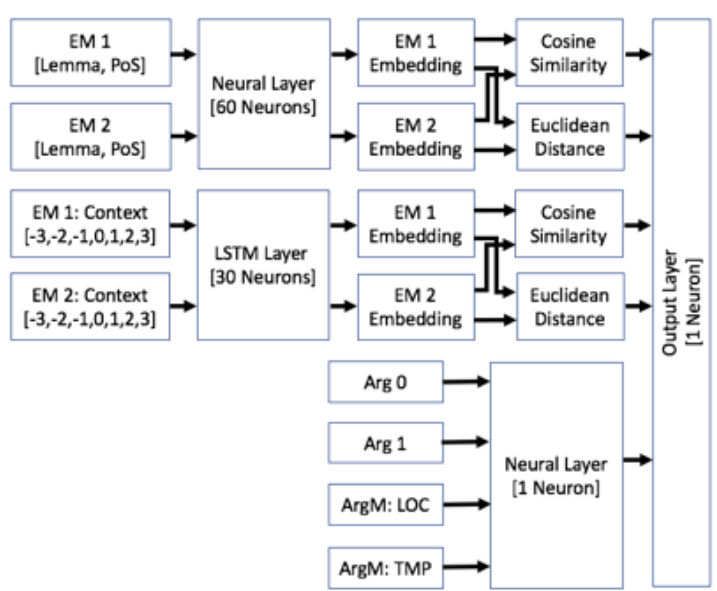

(b)

Figure 2: Pairwise Classifiers for resolving (a) Within-Document Coreference Links (b) Cross-Document Coreference Links. EM: event mentions; ArgO, Arg 1, ArgM:LOC, ArgM:TMP: semantic roles.

ther use context events co-occurring in the same sentence as another parameter to perform additional clustering. Subroutine ContextSimilarity $\left(E_{1}, E_{2}, \Theta_{\mathcal{C D}}\right)$ generates a context vector $(\mathcal{C V})$ for each event cluster and check whether cosine similarity between context vectors of two clusters $E_{1}$ and $E_{2}\left(\cos \left(\overrightarrow{\mathcal{V}}^{1}, \mathcal{C} \overrightarrow{\mathcal{V}}^{2}\right)\right)$ is above 0.7 . Specifically, we define context clusters for an event mention as the different event clusters that have event mentions co-occurring in the same sentence. Then the context vector of an event cluster has an entry for each of its context clusters, with the value to be the number of sentences where event mentions from the two clusters co-occur. This subroutine also makes merges based on the CD confidence score using the same lower threshold of 0.8 .

\section{Distinguishing WD and CD Merging}

We implement two distinct pairwise classifiers to effectively utilize the distributional variations in WD and CD clusters. The first classifier (WD) is used for calculating a similarity between two event mentions within a document and recognizing coreferent event mention pairs. The second classifier (CD) is used for calculating a similarity between two event mentions across two documents and then identifying coreferent event mention pairs across documents. Both classifiers were implemented as neural nets (Chollet, 2015). The architectures of the two classifiers are shown in Figure 2.

WD Classifier: the neural network based WD classifier essentially inherits the features that have been shown effective in previous event coreference studies (Ahn, 2006; Chen et al., 2009), including both features for event words and features for their arguments. Specifically, the classifier includes a common neural layer shared by two event mentions to embed event lemma and partsof-speech features. Then the classifier calculates cosine similarity and euclidean distance between two event embeddings, one per event mention. In addition, the classifier includes a neural layer component to embed event arguments that are overlapped between the two event mentions. Its output layer takes the calculated cosine similarity and euclidean distance between event mention embeddings as well as the embedding of the overlapped event arguments as input, and output a confidence score to indicate the similarity of the two event mentions.

CD Classifier: the CD classifier mimics the WD classifier except that the CD classifier contains an additional LSTM layer (Hochreiter and Schmidhuber, 1997) to embed context words. The LSTM layer is shared by both event mentions in order to calculate context word embeddings for both event mentions. Specifically, three words to each side of an event word together with the event word itself are used to calculate the context embedding for each event mention. The classifier then calculates cosine similarity and euclidean distance between two context embeddings as well. The output neural net layer will take two sets of cosine similarity and euclidean distance scores that have been calculated w.r.t. context embed- 
dings and event word embeddings, as well as the embedding of the overlapped event arguments as input, and further calculate a confidence score indicating the similarity of two event mentions across documents.

\subsection{Characteristics of WD and CD Event Linking}

In order to further understand characteristics of within- and cross-document event linking, we trained two classifiers having the same CD classifier architecture (Figure 2(b)) but with different sets of event pairs, within-document or crossdocument event pairs, then analyzed the impacts of features on each type of event linking by comparing the neural net learned weights for each feature. Table 1 shows the comparisons of feature weights.

\begin{tabular}{|l|l|l|}
\hline Features & WD & CD \\
\hline Event Word Embedding: Euc & 1.017 & 0.207 \\
Event Word Embedding: Cos & 1.086 & 1.142 \\
Context Embedding: Euc & 0.038 & 0.422 \\
Context Embedding: Cos & 0.004 & 3.910 \\
Argument Embedding & 0.349 & 3.270 \\
\hline
\end{tabular}

Table 1: Comparisons of Feature Weights Learned Using In-doc or Cross-doc Coreferent Event Pairs, Euc: Euclidean Distance, Cos: Cosine Similarity

We can see that within-document event linking mainly relies on the euclidean distance and cosine similarity scores calculated using event word features, with a reasonable amount of weight assigned to overlapped arguments' embedding as well. However, only very small weights were assigned to the similarity and distance scores calculated using context embeddings. In contrast, in the classifier trained with cross-doc coreferent event mention pairs, the highest weight was assigned to the cosine similarity score calculated using context embeddings of two event mentions. Additionally, both the cosine similarity score calculated using event word embeddings and the overlapped argument features were assigned high weights as well. The comparisons clearly demonstrate the significantly different nature of WD and CD event coreference.

\subsection{Neural Net Classifiers and Training}

In both WD and CD classifiers, we use neural network layer with 60 neurons for embedding event word features and another layer with 1 neuron for embedding argument features. Additionally, in CD classifier, we use an LSTM layer with 30 neurons to embed context features. Dropout of 0.25 was applied to both the event word neural net layer and the context layer. We used sigmoid activation function for the dense layers and tanh activation for the LSTM layer. We used 300dimensional word embeddings and one hot $37^{4}$ dimensional pos tag embeddings in all our experiments. Therefore, input to word embedding layer is a 337-dimensional vector and to LSTM layer is $300 * 7$ dimensional vectors.

We train both classifiers using the ECB+ corpus (Cybulska and Vossen, 2014b,a). We train the WD classifier using all pairs of WD event mentions that are in an annotated event chain as positive instances and using all pairs of WD event mentions that are not in an annotated event chain as negative instances. However, there are significantly more $\mathrm{CD}$ coreferent event mention pairs annotated in the ECB+ corpus, therefore, we randomly sampled $70 \%$ of all the CD coreferent event mention pairs as positive instances and randomly sampled from non-coreferent CD event mention pairs as negative instances. Specifically, number of negative instances are kept 5 times of positive instances.

Note that the pairwise classifiers will be used throughout the iterative merging stage. However, after each merge, argument propagation is conducted to enrich features for each event mention in the merged cluster and the number of arguments of an event mention will grow after several merges. In order to account for the growing number of arguments in iterative merging, we augment arguments for each event mention in training instances with arguments derived from other event mentions in the same pair. The augmenting was performed randomly for only $50 \%$ of event mentions.

\section{Evaluation}

We perform all the experiments on the ECB+ corpus (Cybulska and Vossen, 2014b,a), which is an extension to the earlier EventCorefBank (ECB) (Bejan and Harabagiu, 2010) dataset. We have adopted the settings used in Yang et al. (2015). We divide the dataset into training set (topics 1-20), validation set (topics 21-23) and test

\footnotetext{
${ }^{4}$ Corresponding to the unique 36 POS tags based on the Stanford POS tagger (Toutanova et al., 2003) and an additional 'padding'.
} 
set (topics 24-43). Table 2 shows the distribution of the corpus.

\begin{tabular}{|l|l|l|l|l|}
\hline & Train & Dev & Test & Total \\
\hline \#Documents & 462 & 73 & 447 & 982 \\
\#Sentences & 7,294 & 649 & 7,867 & 15,810 \\
\#Event Mentions & 3,555 & 441 & 3,290 & 7,286 \\
\#CD Chains & 687 & 47 & 486 & 1,220 \\
\#WD Chains & 2,499 & 316 & 2,137 & 4,952 \\
Avg. WD chain length & 2.835 & 2.589 & 2.553 & 2.686 \\
Avg. CD chain length & 5.17 & 9.39 & 6.77 & 5.98 \\
\hline
\end{tabular}

Table 2: $\mathrm{ECB}+$ Corpus Statistics.

We used event mentions identified by CRF based event extractor used in Yang et al. (2015) and extracted event arguments by applying state-of-the-art semantic role labeling system (SwiRL (Surdeanu et al., 2007)). In addition, we used the Stanford parser (Chen and Manning, 2014) for generating dependency relations, partsof-speech tags and lemmas. We use pre-trained Glove vectors (Pennington et al., 2014) for word $^{5}$ representation and one-hot vectors for parts-ofspeech tags.

We evaluate our model using four commonly adopted event coreference evaluation metrics, namely, MUC (Vilain et al., 1995), $\mathbf{B}^{\mathbf{3}}$ (Bagga and Baldwin, 1998), $\mathbf{C E A F}_{\mathbf{e}}$ (Luo, 2005) and CoNLL F1 (Pradhan et al., 2014). We used the publicly available official implementation of revised coreference scorer (v8.01) ${ }^{6}$

\subsection{Baseline Systems}

We compare our iterative event coreference resolution model with five baseline systems.

LEMMA: The Lemma match baseline links event mentions within- or cross- documents which have the same lemmatized head word. It is often considered a strong baseline for this task.

HDDCRP (Yang et al., 2015): The second baseline is the supervised Hierarchical Distance Dependent Bayesian Model, the most recent event coreference system evaluated on the same ECB+ dataset. This model uses distances between event mentions, generated using a feature-rich learnable distance function, as Bayesian priors for single pass non-parametric clustering.

HDP-LEX ${ }^{7}$ : A reimplementation of the unsupervised hierarchical bayesian model by Bejan

\footnotetext{
${ }^{5}$ Trained on 840 billion tokens of Common Crawl data, http://nlp.stanford.edu/projects/glove/

${ }^{6} \mathrm{https}$ ://github.com/conll/reference-coreference-scorers

${ }^{7}$ The results were taken from the paper Yang et al. (2015).
}

and Harabagiu (2010, 2014).

Agglomerative $^{7}$ : A Reimplementation of twostep agglomerative clustering model, WD clustering followed by CD clustering (Chen et al., 2009).

We have trained our systems using the same $\mathrm{ECB}+$ dataset and the same set of event mentions as these prior systems.

\subsection{Our Systems}

We evaluate several variation systems of our proposed model.

Common Classifier (WD or CD): the system implementing only the first stage of iterative WD $\&$ CD merging. In addition, the same neural net classifier with the architecture as shown in Figure 2(a) (the WD classifier) or in Figure 2(b) (the CD classifier) was applied for both WD and CD merging. The neural net classifiers were trained using all coreferent event mention pairs including both within-document and cross-document ones.

WD and CD Classifiers: distinguishes WD from $\mathrm{CD}$ merges by using two distinct classifiers (Figure 2(a), 2(b)) in the first stage of the algorithm.

+ 2nd Order Relations: after iterative WD and $\mathrm{CD}$ merges within each individual chain as suggested by pairwise classifiers (the first stage), further merges (the second stage) were conducted leveraging second order event inter-dependencies across event chains.

\subsection{Results}

Table 3 shows the comparison results for both within-document and cross-document event coreference resolution. In the first stage of iterative merging, using two distinct WD and CD classifiers for corresponding WD and CD merges yields clear improvements for both WD and CD event coreference resolution tasks, compared with using one common classifier for both types of merges. In addition, the second stage of iterative merging further improves both WD and CD event coreference resolution performance stably by leveraging second order event inter-dependencies. The improvements are consistent when measured using various coreference resolution evaluation metrics.

Our full model achieved more than $8 \%$ of improvements when compared with the lemma matching baseline, using the CoNLL F1-score for both WD and CD coreference resolution tasks. Furthermore, it outperforms state-of-the-art HDDCRP model for both WD and CD event coreference resolution by $2.1 \%$ and $4.9 \%$ respectively. 


\begin{tabular}{|c|c|c|c|c|c|c|c|c|c|c|}
\hline \multicolumn{11}{|c|}{ Cross-Document Coreference Results } \\
\hline & \multicolumn{3}{|l|}{$B^{3}$} & \multicolumn{3}{|c|}{ MUC } & \multicolumn{3}{|c|}{$C E A F E_{e}$} & \multirow{2}{*}{$\begin{array}{l}\text { CoNLL } \\
\text { F1 }\end{array}$} \\
\hline & $\mathrm{R}$ & $\mathrm{P}$ & F1 & $\mathrm{R}$ & $\mathrm{P}$ & F1 & $\mathrm{R}$ & $\mathrm{P}$ & $\mathrm{F} 1$ & \\
\hline LEMMA & 39.5 & 73.9 & 51.4 & 58.1 & 78.2 & 66.7 & 58.9 & 37.5 & 46.2 & 54.8 \\
\hline Common Classifier (WD) & 46 & 72.8 & 56.4 & 60.4 & 76.8 & 68.4 & 59.5 & 42.1 & 49.3 & 58 \\
\hline+2 nd Order Relations & 48.8 & 72.1 & 58.2 & 61.8 & 78.9 & 69.3 & 59.3 & 44.1 & 50.6 & 59.4 \\
\hline Common Classifier (CD) & 44.9 & 64.7 & 53 & 66.1 & 66.4 & 66.2 & 51.9 & 46.4 & 49 & 56.1 \\
\hline+2 nd Order Relations & 52.2 & 58.4 & 55.1 & 70.4 & 66.2 & 68.3 & 54.1 & 45.2 & 49.2 & 57.5 \\
\hline WD \& CD Classifiers & 49 & 71.9 & 58.3 & 63.8 & 78.9 & 70.6 & 59.3 & 48.1 & 53.1 & 60.7 \\
\hline + 2nd Order Relations (Full Model) & 56.2 & 66.6 & 61 & 67.5 & 80.4 & 73.4 & 59 & 54.2 & 56.5 & 63.6 \\
\hline HDDCRP Yang et al. (2015) & 40.6 & 78.5 & 53.5 & 67.1 & 80.3 & 73.1 & 68.9 & 38.6 & 49.5 & 58.7 \\
\hline HDP-LEX Beja & 43.7 & 65.6 & 52.5 & 63.5 & 75.5 & 69.0 & 60.2 & 34.8 & 44.1 & 55.2 \\
\hline Agglomerative Chen et al. (2009) & 40.2 & 73.2 & 51.9 & 59.2 & 78.3 & 67.4 & 65.6 & 30.2 & 41.1 & 53.6 \\
\hline \multicolumn{11}{|c|}{ Within-Document Coreference Results } \\
\hline LEMMA & 56.8 & 80.9 & 66.7 & 35.9 & 76.2 & 48.8 & 67.4 & 62.9 & 65.1 & 60.2 \\
\hline Common Classifi & 59.7 & 80.5 & 68.6 & 44.6 & 75 & 55.9 & 68.2 & 67.7 & 67.9 & 64.2 \\
\hline+2 nd Order Relations & 62.7 & 79.4 & 70 & 50.3 & 75.2 & 60.3 & 68.6 & 70.5 & 69.5 & 66.6 \\
\hline Common Classifier (CD) & 65.2 & 67.1 & 66.1 & 47.6 & 53.9 & 50.5 & 69.2 & 62.1 & 65.5 & 60.7 \\
\hline + 2nd Order Relations & 66.9 & 69.1 & 68 & 56.7 & 55.1 & 55.9 & 70.4 & 63.6 & 66.8 & 62.8 \\
\hline WD \& CD classifiers & 63.8 & 79.9 & 70.9 & 51.6 & 75.3 & 61.2 & 68.6 & 70.5 & 69.5 & 67.2 \\
\hline + 2nd Order Relations (Full Model) & 69.2 & 76 & 72.4 & $\mathbf{5 8 . 5}$ & 67.3 & 62.6 & 67.9 & 76.1 & 71.8 & 68.9 \\
\hline HDDCRP Yang et al. (2015) & 67.3 & 85.6 & 75.4 & 41.7 & 74.3 & 53.4 & 79.8 & 65.1 & 71.7 & 66.8 \\
\hline HDP-LEX Bejan and Harabagiu (2010) & 67.6 & 74.7 & 71.0 & 39.1 & 50.0 & 43.9 & 71.4 & 66.2 & 68.7 & 61.2 \\
\hline Agglomerative Chen et al. (2009) & 67.6 & 80.7 & 73.5 & 39.2 & 61.9 & 48.0 & 76.0 & 65.6 & 70.4 & 63.9 \\
\hline
\end{tabular}

Table 3: Within- and cross-document event coreference result on ECB+ Corpus.

\section{Discussion and Analysis}

\begin{tabular}{|l|l|l|l|l|}
\hline \multicolumn{5}{|c|}{ Cross-Document Coreference Results } \\
\hline$F_{\text {measure }}$ & $B^{3}$ & MUC & $C E A F E_{e}$ & CoNLL \\
\hline 1 Iteration & 56 & 69.3 & 50.3 & 58.5 \\
2 Iterations & 57.9 & 69.9 & 52.4 & 60.1 \\
3 Iterations & 58.3 & 70.6 & 53.1 & 60.7 \\
\hline \hline \multicolumn{5}{|c|}{ Within-Document Coreference Results } \\
\hline 1 Iteration & 69.7 & 55.8 & 68.8 & 64.8 \\
2 Iterations & 70.2 & 60.3 & 69.4 & 66.6 \\
3 Iterations & 70.9 & 61.2 & 69.5 & 67.2 \\
\hline
\end{tabular}

Table 4: Per-iteration Performance Analysis for the First Stage of Iterative WD \& CD Merging.

Stage I: The first stage of our algorithm, iterative WD and CD merging, went for three iterations (See Table 4). Our analysis of merges in each iteration shows that most of the merges in the initial iteration are between event mentions with the same lemma or shared arguments. In the second and third iterations, more merges were between event mentions with synonymous lemmas or shared arguments that have been accumulated in previous iterations. Example merges between synonymous event mentions include (nominate, nominations), (die, death), (murder, killing), (hit, strike), (attack, bomb) etc.

Stage II: It is even more intriguing to discuss the clusters that were merged in stage 2 of merging, that leverages second order event interdependencies across event chains. We found that al- most all of the 81 merges happening in the second stage are between event mentions that are quite dissimilar including (take over, replace), (unveil, announce), (win, victory, comeback), (downtime, problem, outage), (cut, damage), (spark, trigger) etc. Most interestingly, two event pairs which are antonymous to each other, (win, beat) and (defeat, victory), were also correctly merged.

Errors: while our iterative algorithm has gradually resolved coreference relations between event mentions that are synonyms or distant by surface forms, many coreference links were overlooked and many unrelated events were wrongly predicted as coreferential. We analyzed our system's final predictions in order to identify the most common sources of errors.

Missed Coreference Links: We found that many event mentions have few or no argument in their local context, and our event coreference resolution system often failed to link these event mentions with their coreferential mentions. For instance, in the following event mention pairs that were overlooked by the system, (operations, raids), (operations, sweep), (suicide, hang), (prosecution, jail), and (participating, role), one or both event mentions do not have an argument in their local context. This is mainly because the base WD and CD classifiers heavily rely on features extracted from the local context of two event men- 
tions, including event words and event arguments, in resolving the coreference relation. For these event mentions having few arguments identified, the iterative algorithm may get stuck from the beginning.

While it is a grand challenge to further resolve coreferential relations between event mentions that do not have sufficient local features, these missed coreference links easily break a long and influential event chain into several sub-chains, which makes event coreference resolution results less useful for many potential applications, such as text summarization.

Wrongly Predicted Coreference Links: The majority of this type of errors are between noncoreferent event mentions that have the same lemma. This is especially common among reporting event mentions and light verb mentions. For instance, we found that 24 non-coreferent event clusters corresponding to reporting events, e.g., said, told and reported, and 13 non-coreferent clusters corresponding to light verbs, e.g., take, give and get, were incorrectly merged by the system.

\section{Conclusions and Future Work}

We presented a novel approach for event coreference resolution that extensively exploits event inter-dependencies between event mentions in the same chain and event mentions across chains. The approach iteratively conducts WD and CD merges followed by further merges leveraging second order event inter-dependencies across chains. We further distinguish WD and CD merges using two distinct classifiers that capture differences of within- and cross-document event clusters in feature distributions. Our system was shown effective in both WD and CD event coreference and has outperformed the previous best event coreference system in both tasks.

Note that our approach is flexible to incorporate different strategies for conducting WD and $\mathrm{CD}$ merges. In the future, we plan to continue to investigate the distinct characteristics of WD and $\mathrm{CD}$ coreferent event mentions in order to further improve event coreference performance. Especially, we are interested in including additional discourse-level features for improving WD coreference merge performance, such as, features indicating the distance between two event mentions in a document.

\section{Acknowledgments}

We want to thank our anonymous reviewers for providing insightful review comments.

\section{References}

David Ahn. 2006. The stages of event extraction. In Proceedings of the Workshop on Annotating and Reasoning about Time and Events, pages 1-8. Association for Computational Linguistics.

James Allan, Jaime G Carbonell, George Doddington, Jonathan Yamron, and Yiming Yang. 1998. Topic detection and tracking pilot study final report.

Amit Bagga and Breck Baldwin. 1998. Algorithms for scoring coreference chains. In The first international conference on language resources and evaluation workshop on linguistics coreference, volume 1, pages 563-566.

Cosmin Adrian Bejan and Sanda Harabagiu. 2010. Unsupervised event coreference resolution with rich linguistic features. In Proceedings of the 48th Annual Meeting of the Association for Computational Linguistics, pages 1412-1422. Association for Computational Linguistics.

Cosmin Adrian Bejan and Sanda Harabagiu. 2014. Unsupervised event coreference resolution. Computational Linguistics, 40(2):311-347.

Lars Buitinck, Gilles Louppe, Mathieu Blondel, Fabian Pedregosa, Andreas Mueller, Olivier Grisel, Vlad Niculae, Peter Prettenhofer, Alexandre Gramfort, Jaques Grobler, Robert Layton, Jake VanderPlas, Arnaud Joly, Brian Holt, and Gaël Varoquaux. 2013. API design for machine learning software: experiences from the scikit-learn project. In ECML PKDD Workshop: Languages for Data Mining and Machine Learning, pages 108-122.

Danqi Chen and Christopher D Manning. 2014. A fast and accurate dependency parser using neural networks. In EMNLP, pages 740-750.

Zheng Chen and Heng Ji. 2009. Graph-based event coreference resolution. In Proceedings of the 2009 Workshop on Graph-based Methods for Natural Language Processing, pages 54-57. Association for Computational Linguistics.

Zheng Chen, Heng Ji, and Robert Haralick. 2009. A pairwise event coreference model, feature impact and evaluation for event coreference resolution. In Proceedings of the workshop on events in emerging text types, pages 17-22. Association for Computational Linguistics.

François Chollet. 2015. Keras. https://github. com/fchollet/keras.

Kevin Clark and Christopher D Manning. 2015. Entitycentric coreference resolution with model stacking. 
Kevin Clark and Christopher D Manning. 2016. Improving coreference resolution by learning entitylevel distributed representations.

Agata Cybulska and Piek Vossen. 2014a. Guidelines for ecb+ annotation of events and their coreference. Technical report, Technical report, Technical Report NWR-2014-1, VU University Amsterdam.

Agata Cybulska and Piek Vossen. 2014b. Using a sledgehammer to crack a nut? lexical diversity and event coreference resolution. In $L R E C$, pages 4545 4552 .

Agata Cybulska and Piek Vossen. 2015a. Translating granularity of event slots into features for event coreference resolution. In Proceedings of the $3 \mathrm{rd}$ Workshop on EVENTS at the NAACL-HLT, pages 110.

Agata Cybulska and Piek Vossen. 2015b. bag of events approach to event coreference resolution. supervised classification of event templates. IJCLA, page 11.

Naomi Daniel, Dragomir Radev, and Timothy Allison. 2003. Sub-event based multi-document summarization. In Proceedings of the HLT-NAACL 03 on Text summarization workshop-Volume 5, pages 9-16. Association for Computational Linguistics.

Sepp Hochreiter and Jürgen Schmidhuber. 1997. Long short-term memory. Neural computation, 9(8):1735-1780.

Kevin Humphreys, Robert Gaizauskas, and Saliha Azzam. 1997. Event coreference for information extraction. In Proceedings of a Workshop on Operational Factors in Practical, Robust Anaphora Resolution for Unrestricted Texts, pages 75-81. Association for Computational Linguistics.

Heeyoung Lee, Marta Recasens, Angel Chang, Mihai Surdeanu, and Dan Jurafsky. 2012. Joint entity and event coreference resolution across documents. In Proceedings of the 2012 Joint Conference on Empirical Methods in Natural Language Processing and Computational Natural Language Learning, pages 489-500. Association for Computational Linguistics.

Zhengzhong Liu, Jun Araki, Eduard H Hovy, and Teruko Mitamura. 2014. Supervised withindocument event coreference using information propagation. In $L R E C$, pages 4539-4544.

Jing Lu, Deepak Venugopal, Vibhav Gogate, and Vincent Ng. 2016. Joint inference for event coreference resolution.

Xiaoqiang Luo. 2005. On coreference resolution performance metrics. In Proceedings of the conference on Human Language Technology and Empirical Methods in Natural Language Processing, pages 25-32. Association for Computational Linguistics.
James Mayfield, David Alexander, Bonnie J Dorr, Jason Eisner, Tamer Elsayed, Tim Finin, Clayton Fink, Marjorie Freedman, Nikesh Garera, Paul McNamee, et al. 2009. Cross-document coreference resolution: A key technology for learning by reading. In AAAI Spring Symposium: Learning by Reading and Learning to Read, volume 9, pages 65-70.

Srini Narayanan and Sanda Harabagiu. 2004. Question answering based on semantic structures. In Proceedings of the 20th international conference on Computational Linguistics, page 693. Association for Computational Linguistics.

Jeffrey Pennington, Richard Socher, and Christopher D Manning. 2014. Glove: Global vectors for word representation. In EMNLP, volume 14, pages 15321543.

Sameer Pradhan, Xiaoqiang Luo, Marta Recasens, Eduard Hovy, Vincent Ng, and Michael Strube. 2014. Scoring coreference partitions of predicted mentions: A reference implementation. In Proceedings of the 52nd Annual Meeting of the Association for Computational Linguistics (Volume 2: Short Papers), pages 30-35, Baltimore, Maryland. Association for Computational Linguistics.

Sameer Singh, Karl Schultz, and Andrew McCallum. 2009. Bi-directional joint inference for entity resolution and segmentation using imperatively-defined factor graphs. Machine Learning and Knowledge Discovery in Databases, pages 414-429.

Mihai Surdeanu, Lluís Màrquez, Xavier Carreras, and Pere R Comas. 2007. Combination strategies for semantic role labeling. Journal of Artificial Intelligence Research, 29:105-151.

K. Toutanova, D. Klein, C. Manning, and Y. Singer. 2003. Feature-Rich Part-of-Speech Tagging with a Cyclic Dependency Network. In Proceedings of HLT-NAACL 2003.

Marc Vilain, John Burger, John Aberdeen, Dennis Connolly, and Lynette Hirschman. 1995. A modeltheoretic coreference scoring scheme. In Proceedings of the 6th conference on Message understanding, pages 45-52. Association for Computational Linguistics.

Sam Wiseman, Alexander M Rush, and Stuart M Shieber. 2016. Learning global features for coreference resolution. In Proceedings of NAACL-HLT, pages 994-1004.

Bishan Yang, Claire Cardie, and Peter Frazier. 2015. A hierarchical distance-dependent bayesian model for event coreference resolution. Transactions of the Association for Computational Linguistics, 3:517528.

Tongtao Zhang, Hongzhi Li, Heng $\mathrm{Ji}$, and ShihFu Chang. 2015. Cross-document event coreference resolution based on cross-media features. In EMNLP, pages 201-206. 